\title{
Generalized entropic criterion for separability
}

\author{
R. Rossignoli and N. Canosa \\ Departamento de Física, Universidad Nacional de La Plata, Casilla de Correo 67, La Plata (1900), Argentina
}

(Received 21 April 2002; published 11 October 2002)

\begin{abstract}
We discuss the entropic criterion for separability of compound quantum systems for general nonadditive entropic forms based on arbitrary concave functions $f$. For any separable state, the generalized entropy of the whole system is shown to be not smaller than that of the subsystems, for any choice of $f$, providing thus a necessary criterion for separability. Nevertheless, the criterion is not sufficient and examples of entangled states with the same property are provided. This entails, in particular, that the conjecture about the positivity of the conditional Tsallis entropy for all $q$, a more stringent requirement than the positivity of the conditional von Neumann entropy, is actually a necessary but not sufficient condition for separability in general. The direct relation between the entropic criterion and the largest eigenvalues of the full and reduced density operators of the system is also discussed.
\end{abstract}

DOI: $10.1103 /$ PhysRevA.66.042306

PACS number(s): 03.67.-a, 03.65.Ud, 05.30.-d

The concept of quantum entanglement [1] has aroused great interest in recent years, due to its deep implications for quantum computation [2], quantum cryptography [3], and quantum teleportation [4]. The relation between entropy and quantum entanglement has also attracted attention from several authors [5-15]. It is well known, for instance, that the von Neumann entropy of a compound quantum system may be larger or smaller than that of a subsystem $[16,17]$. However, if the system is in a separable (i.e., unentangled) state, the von Neumann entropy of the whole system is not smaller than that of a subsystem $[5,6]$. Unfortunately, the converse is not true, i.e., the same may occur when the system is in an inseparable (i.e., entangled) state, so that this entropy provides only a necessary test for separability. The von Neumann based criterion is actually rather weak, being less stringent than other equally simple necessary conditions $[5,18,19]$. As discussed in $[7,14,15]$, the von Neumann entropy is in fact not a good entanglement indicator even in those cases where entanglement is fully determined by the eigenvalues of the density operator $\rho$.

These facts suggest consideration of other information measures which could capture more effectively the effects associated with the separability or inseparability of a compound quantum system. In particular, it has been shown that nonadditive information measures like that of Tsallis [20] do provide more stringent conditions for separability $[11,12]$. Moreover, this entropy depends on a parameter $q$ which can be optimized. In fact, for $q \rightarrow \infty$, necessary and sufficient conditions for separability were obtained with this entropy for some important classes of states, like Werner states for $n$ qubits and also $n$ qudits [11,12]. In other situations [13], entanglement was detected, however, at finite values of $q$, rather than in the $q \rightarrow \infty$ limit. Hence, the questions arise of whether this entropy could provide a necessary and/or sufficient test in general and whether other information measures could lead to the same result.

In this article we will examine more general entropic forms based on arbitrary concave functions, which include as particular cases the von Neumann and Tsallis entropies. We will show that any of these forms provide necessary conditions for separability, which are not sufficient in general. It will also become clear why the Tsallis form provides necessary and sufficient conditions for Werner states in the $q$ $\rightarrow \infty$ limit, and why it is not so in other situations. Finally, other entropic forms providing similar results are given.

Let us consider a quantum system described by a density operator $\rho$. We will examine the general entropic forms [15]

$$
S_{f}(\rho)=\operatorname{Tr} f(\rho)=\sum_{i} f\left(p_{i}\right)
$$

where $f$ is a smooth concave function $\left[f^{\prime}(p)\right.$ decreasing for $p \in(0,1)]$ satisfying $f(0)=f(1)=0$, and $p_{i}, i=1, \ldots, n$, are the eigenvalues of $\rho\left(\Sigma_{i} p_{i}=1\right)$. We assume a finite dimension $n$. The von Neumann entropy is recovered for

$$
f(p)=-k p \ln p
$$

with $k>0$, while the Tsallis entropy corresponds to [20]

$$
f(p)=\left(p-p^{q}\right) /(q-1), \quad q>0,
$$

which approaches $-p \ln p$ for $q \rightarrow 1$. The generalized entropies (1) satisfy most basic properties of the conventional entropy, except those related to additivity. In particular, $S_{f}(\rho)$ $\geqslant 0$, with $S_{f}(\rho)=0$ if and only if the system is in a pure state $\left(\rho^{2}=\rho\right)$, while its maximum is attained for the fully mixed state $\rho=I / n$ [21]. Concavity of $f$ ensures concavity of $S_{f}(\rho) \quad[17]\left[S_{f}\left(\Sigma_{j} q_{j} \rho_{j}\right) \geqslant \Sigma_{j} q_{j} S_{f}\left(\rho_{j}\right)\right.$ for $0 \leqslant q_{j} \leqslant 1, \quad \sum_{j} q_{j}$ $=1]$. It can be shown $[21,15]$ that if $\left[p f^{\prime \prime}(p)\right]^{\prime} \leqslant 0(\geqslant 0)$, then $S_{f}$ is sub-(super)additive, i.e., $S_{f}\left(\rho_{A} \otimes \rho_{B}\right)-S_{f}\left(\rho_{A}\right)$ $-S_{f}\left(\rho_{B}\right) \leqslant 0(\geqslant 0)$. The condition $\left[p f^{\prime \prime}(p)\right]^{\prime}=0$ determines in fact Eq. (2). The Tsallis entropy is, accordingly, sub(super)additive for $q>1(q<1)$.

A fundamental property of the forms (1) which will be employed in this work, and which justifies their use as information measures, is that if $\rho$ is more mixed than a density operator $\rho^{\prime}$, then

$$
S_{f}(\rho) \geqslant S_{f}\left(\rho^{\prime}\right)
$$


for any $f$ of the previous form [17]. Labeling the eigenvalues of $\rho$ and $\rho^{\prime}$ in decreasing order, i.e., $p_{1} \geqslant p_{2} \geqslant \ldots \geqslant p_{n}, \quad \rho$ is said to be more mixed (or disordered) than $\rho^{\prime}$ if

$$
\mathcal{S}_{i}=\sum_{j=1}^{i} p_{j} \leqslant \mathcal{S}_{i}^{\prime}=\sum_{j=1}^{i} p_{j}^{\prime}, \quad i=1, \ldots, n-1,
$$

i.e., if $p_{1} \leqslant p_{1}^{\prime}, p_{1}+p_{2} \leqslant p_{1}^{\prime}+p_{2}^{\prime}$, etc. (for $i=n, \mathcal{S}_{n}=\mathcal{S}_{n}^{\prime}$ $=1)$. Mathematically, this states that the set of probabilities $\left(p_{1}, \ldots, p_{n}\right)$ is majorized by $\left(p_{1}^{\prime}, \ldots, p_{n}^{\prime}\right)$. Equation (4) can be immediately derived by writing $p_{i}=\mathcal{S}_{i}-\mathcal{S}_{i-1}$ in Eq. (1), with $\mathcal{S}_{0}=0 . S_{f}(\rho)$ is then a decreasing function of $\mathcal{S}_{i}$ for $1 \leqslant i \leqslant n-1$, as $\partial S_{f} / \partial \mathcal{S}_{i}=f^{\prime}\left(p_{i}\right)-f^{\prime}\left(p_{i+1}\right) \leqslant 0$ if $p_{i} \geqslant p_{i+1}$ and $f$ is concave [Eq. (4) follows then from the mean value theorem; note that the allowed values of $\mathcal{S}_{i}$ form a convex set defined by $\mathcal{S}_{i} \leqslant \mathcal{S}_{i+1}, \quad \mathcal{S}_{i} \geqslant\left(\mathcal{S}_{i-1}+\mathcal{S}_{i+1}\right) / 2$, with $\mathcal{S}_{0}=0, \mathcal{S}_{n}$ $=1]$.

Moreover, it can be shown [17] that $\rho$ is more mixed than $\rho^{\prime}$ if and only if $\operatorname{Tr} f(\rho) \geqslant \operatorname{Tr} f\left(\rho^{\prime}\right)$ for any concave $f$, i.e., if and only if Eq. (4) holds $\forall f$ of the previous form [the conditions $f(0)=f(1)=0$ fix just an arbitrary linear term ap $+b$ that can be added to $f$ without affecting concavity or Eq. (4)]. If the dimensions of $\rho$ and $\rho^{\prime}$ differ, we may apply the same definition of "more mixed" by adding zero eigenvalues to the density with the smallest dimension, which leaves $S_{f}$ unchanged.

Let us consider now a system composed of two subsystems $A$ and $B$. The quantity

$$
S_{f}^{A}(\rho) \equiv S_{f}(\rho)-S_{f}\left(\rho_{A}\right)=\operatorname{Tr} f(\rho)-\operatorname{Tr}_{A} f\left(\rho_{A}\right),
$$

where $\rho_{A}=\operatorname{Tr}_{B} \rho$ is the reduced density matrix of system $A$ and $\operatorname{Tr}=\operatorname{Tr}_{A} \operatorname{Tr}_{B}$, plays the role of a conditional entropy. In the von Neumann case, Eq. (6) becomes the usual conditional entropy [17],

$$
S_{f}^{A}(\rho)=S(B \mid A)=-\operatorname{Tr} \rho\left[\ln \rho-\ln \rho_{A} \otimes I_{B}\right],
$$

whereas, in the Tsallis case, it is proportional to the $q$-conditional entropy defined in $[11,12], \quad S_{q}(B \mid A)$ $=S_{f}^{A}(\rho) / \operatorname{Tr} \rho_{A}^{q}$.

For a discrete classical system described by a joint probability distribution $p_{i j}$, Eq. (6) is always non-negative, i.e.,

$$
\sum_{i, j} f\left(p_{i j}\right)-\sum_{i} f\left(p_{i}\right) \geqslant 0, \quad p_{i}=\sum_{j} p_{i j}
$$

since for any concave $f$ satisfying $f(0)=0$ we have $f(p$ $+q) \leqslant f(p)+f(q)$ if $p \geqslant 0, q \geqslant 0$ (it may also be seen that the set of probabilities $\left\{p_{i j}\right\}$ is more mixed than $\left.\left\{p_{i}\right\}\right)$. This implies that $S_{f}^{A}(\rho) \geqslant 0$ for any uncorrelated density $\rho=\rho_{A}$ $\otimes \rho_{B}$ (i.e., $p_{i j}=p_{i}^{A} p_{j}^{B}$ ) as well as for any density diagonal in a basis of product states $\left(\rho=\Sigma_{i, j} p_{i j}\left|i_{A} j_{B}\right\rangle\left\langle i_{A} j_{B}\right|\right)$. Nevertheless, in the general quantum case, $S_{f}^{A}(\rho)$ may of course be negative. In particular, for a pure state $\rho=|\Psi\rangle\langle\Psi|, S_{f}(\rho)$ $=0$ and the positive eigenvalues of $\rho_{A}$ and $\rho_{B}$ are identical [17], whence

$$
S_{f}^{A}(\rho)=-S_{f}\left(\rho_{A}\right)=-S_{f}\left(\rho_{B}\right) \leqslant 0 .
$$

For $f(p)=-p \log _{2} p$, this is just the usual definition of the entanglement of a pure state $|\Psi\rangle[22,23]$.

Negative values of $S_{f}^{\mathrm{A}}(\rho)$ are then indicative of distinctive quantum correlations. In particular, for the case (3) it has been conjectured [11-13] that the sign of the difference (6) may provide a criterion for determining the separability of $\rho$ [13]. Let us recall that a mixed state $\rho$ is separable (or classically correlated) if and only if it can be written as a convex combination of uncorrelated densities [24],

$$
\rho=\sum_{\alpha} \omega_{\alpha} \rho_{A}^{\alpha} \otimes \rho_{B}^{\alpha}, \quad 0 \leqslant \omega_{\alpha} \leqslant 1,
$$

with $\Sigma_{\alpha} \omega_{\alpha}=1$. Otherwise it is called entangled or inseparable. For the Tsallis case, it has been shown $[11,12]$ that the criterion $S_{f}^{A}(\rho) \geqslant 0$ leads, for $q \rightarrow \infty$, to the necessary and sufficient condition for separability for some important classes of states, like Werner states. Nevertheless, we will show here that this does not hold in general. In particular, for an entangled state $S_{f}^{A}(\rho)$ and $S_{f}^{B}(\rho)$ may in fact both be positive for any concave $f$ (including the $q \rightarrow \infty$ limit in the Tsallis case), indicating that entanglement cannot always be detected by such entropic criteria (or, in general, by information based on the eigenvalues of $\rho$ and $\rho_{A, B}$ alone). This may occur already for a two-qubit system, where the Peres necessary criterion for separability [18] is known to be sufficient [19], so that the entropic criterion is here weaker than the Peres criterion.

Let us first show that Eq. (6) is indeed positive for any separable $\rho$. A fundamental theorem demonstrated in [25] states that if $\rho$ is separable, then $\rho$ is more mixed than $\rho_{A}$ and $\rho_{B}$ (disorder criterion for separability). Hence, Eq. (4) implies that if $\rho$ is separable, then

$$
S_{f}^{A}(\rho) \geqslant 0
$$

and similarly $S_{f}^{B}(\rho) \geqslant 0$, for any concave $f$ [satisfying $f(0)$ $=0]$. This is in fact an equivalent entropic formulation of the disorder criterion. For a separable state, Eq. (10) will therefore hold $\forall q>0$ in the case (3), implying $\operatorname{Tr} \rho^{q}-\operatorname{Tr}_{A} \rho_{A}^{q}$ $\leqslant 0(\geqslant 0)$ if $q>1(0<q<1)$. Note that this entails $S_{\alpha}(\rho)$ $\geqslant S_{\alpha}\left(\rho_{A}\right) \forall \alpha>0$, where $S_{\alpha}(\rho)=(1-\alpha)^{-1} \ln \operatorname{Tr} \rho^{\alpha}$ is the Rényi entropy $[26,5]$ [which is additive but not of the form (1), and approaches the von Neumann entropy for $\alpha \rightarrow 1]$. The disorder criterion is, however, not sufficient [25], so that Eq. (10) provides in general only a necessary test for separability, as will be explicitly seen below.

For a system of two qubits, Eq. (10) is actually an immediate consequence of the more obvious fact that for any separable state,

$$
p_{1} \leqslant p_{1}^{A},
$$

where $p_{1}\left(p_{1}^{A}\right)$ denotes the largest eigenvalue of $\rho\left(\rho_{A}\right)$. This is so because the difference

$$
\rho_{d}=\rho_{A} \otimes I_{B}-\rho=\sum_{\alpha} \omega_{\alpha} \rho_{A}^{\alpha} \otimes\left(I_{B}-\rho_{B}^{\alpha}\right)
$$


is a non-negative operator if all $\omega_{\alpha} \geqslant 0$ [27]. Hence, denoting by $|i\rangle$ any eigenstate of $\rho$, we have

$$
0 \leqslant\left\langle i\left|\rho_{d}\right| i\right\rangle=\left\langle i\left|\rho_{A} \otimes I_{B}\right| i\right\rangle-p_{i} \leqslant p_{1}^{A}-p_{i},
$$

since $\quad\left\langle i\left|\rho_{A} \otimes I_{B}\right| i\right\rangle \leqslant\left\langle 1_{A} j_{B}\left|\rho_{A} \otimes I_{B}\right| 1_{A} j_{B}\right\rangle=p_{1}^{A}, \quad$ where $\rho_{A}\left|1_{A}\right\rangle=p_{1}^{A}\left|1_{A}\right\rangle$ and $\left|j_{B}\right\rangle$ is any state of $B$. For a two-qubit system, Eq. (11) already implies that $\rho$ is more mixed than $\rho_{A}: \sum_{j=1}^{i} p_{j} \leqslant p_{1}^{A}+p_{2}^{A}=1$ for $i=2,3,4$.

There are two important remarks to make here. First, if $p_{1}>p_{1}^{A}$, the state is certainly entangled, but $\rho_{A}$ is not necessarily more mixed than $\rho$, entailing that $S_{f}^{A}(\rho)$ is not necessarily negative for any $f$. Nevertheless, in the Tsallis case, as well as for any set of entropic functions

$$
f(p)=k\left[p-g_{q}(p)\right]
$$

where $k>0$ and $g_{q}(p)$ is a convex increasing function satisfying $g_{q}(0)=0, g_{q}(1)=1$, and

$$
\lim _{q \rightarrow \infty} g_{q}\left(p^{\prime}\right) / g_{q}(p)=0 \text { if } p^{\prime}<p,
$$

$S_{f}(\rho)$ will be a decreasing function of the largest eigenvalue $p_{1}$ for sufficiently large $q$ and finite dimension $\left[S_{f}(\rho)\right.$ $\approx k\left(1-d_{1} g_{q}\left(p_{1}\right)\right)$ in this limit, with $d_{1}$ the multiplicity of $p_{1}$ ]. Hence, if $p_{1}>p_{1}^{A}, S_{f}^{A}(\rho)$ will become negative for sufficiently large $q$, and the entropic criterion will be able to detect entanglement. In other words, for $q \rightarrow \infty, S_{f}^{A}(\rho)<0$ if and only if $p_{1}>p_{1}^{A}$, which is a sufficient condition for inseparability. Note that Eq. (3) is of the form (14) for $q>1$ and satisfies Eq. (15). Another example is [15]

$$
f(p)=\frac{1}{q}\left[p-\frac{e^{q p}-1}{e^{q}-1}\right],
$$

which is concave $\forall q$, approaches $\frac{1}{2} p(1-p)$ for $q \rightarrow 0[q$ $=2$ case in Eq. (3)] and is of the form (14) for $q>0$.

Nonetheless, and this is the second important remark, there are entangled states for which $p_{1} \leqslant p_{1}^{A}$ and $p_{1}^{B}$, i.e., for which the greatest eigenvalue of $\rho$ remains smaller than that of $\rho_{A}$ and $\rho_{B}$. This may occur already for a system of two qubits, in which case $\rho$ will remain more mixed than $\rho_{A}$ and $\rho_{B}$, and $S_{f}^{A}(\rho), S_{f}^{B}(\rho)$ will both be non-negative for any concave $f$. This type of entanglement will therefore not be detected by the previous entropic criterion.

An example is the state considered in [18],

$$
\rho=x\left|\Psi_{0}\right\rangle\left\langle\Psi_{0}|+(1-x)| \uparrow \uparrow\right\rangle\langle\uparrow \uparrow|, \quad 0 \leqslant x \leqslant 1,
$$

where $\left|\Psi_{0}\right\rangle=(|\uparrow \downarrow\rangle-|\downarrow \uparrow\rangle) / \sqrt{2}$ is the singlet (a maximally entangled state) and $|\uparrow \uparrow\rangle$ a maximally polarized separable state. As shown in [18], the Peres criterion determines that this state is entangled $\forall x>0$ : the partial transpose of $\rho$ (defined as the transposition with respect to the indices of system $A$ ), which is still a density operator if $\rho$ is separable, has always a negative eigenvalue for $x>0$, namely, $\sigma_{1}$ $=\frac{1}{2}[1-x-\sqrt{1-2 x(1-x)}]\left[\sigma_{1}=-x^{2} / 4+O\left(x^{3}\right)\right.$ for $x$ $\rightarrow 0]$.



FIG. 1. Top: The largest eigenvalue $p_{1}$ of $\rho$ and $p_{1}^{A}$ of $\rho_{A}$, for the density (17), as a function of the parameter $x$. The dotted line depicts the lowest eigenvalue $\sigma_{1}$ of the partial transpose of $\rho$. Center: The normalized entropic difference (18) for the Tsallis case (3), at the indicated values of $q$. The curve for $q=1$ corresponds to the von Neumann entropy, in which case $\overline{S_{f}^{A}}=S(B \mid A)$. Bottom: The same quantity for the entropic function (16). The curve for $q=0$ depicts the limit $\overline{S_{f}^{A}}=(1 / 2) \operatorname{Tr}\left[\rho_{A}^{2}-\rho^{2}\right]$. The point where $p_{1}=p_{1}^{A}$ is indicated by $x_{c}$. Both $x$ and the quantities plotted are dimensionless.

However, as the eigenvalues of $\rho$ are $(x, 1-x, 0,0)$, and those of $\rho_{A}$ and $\rho_{B}$ are $(1-x / 2, x / 2)$, the greatest eigenvalue of $\rho\left(p_{1}=x\right.$ for $\left.x>\frac{1}{2}\right)$ is greater than that of $\rho_{A}\left(p_{1}^{A}=1\right.$ $-x / 2$ ) only for $x>x_{c}=2 / 3$ (Fig. 1). Hence, for $0<x<2 / 3$, entanglement will not be detected by $S_{f}^{A, B}(\rho)$, for any $f$. This can also be directly seen from the explicit expression

$$
S_{f}^{A}(x)=f(x)+f(1-x)-[f(x / 2)+f(1-x / 2)] .
$$

Since for a two state system, the entropy $f(p)+f(1-p)$ is a decreasing function of the largest eigenvalue $\left[f^{\prime}(p)-f^{\prime}(1\right.$ $-p)<0$ for $p>1 / 2$ and $f$ concave], in this case $S_{f}^{A}(\rho)<0$ if and only if $p_{1}>p_{1}^{A}$, i.e., $S_{f}^{A}(x)<0$ if and only if $x>2 / 3$, for any $f$. The sign of $S_{f}^{A}(x)$ is independent of the choice of entropic function $f$ in this example, i.e., independent of $q$ in the Tsallis case or in Eq. (16), as shown in Fig. 1. For normalization purposes, we have plotted the quantity

$$
\overline{S_{f}^{A}}(\rho)=S_{f}^{A}(\rho) / \operatorname{Tr} g_{q}\left(\rho_{A}\right),
$$

where $g_{q}(p)=p^{q}$ in the Tsallis case (3) [so that $\overline{S_{f}^{A}}(\rho)$ $\left.=S_{q}(B \mid A)\right]$ and $g_{q}(p)=\left(e^{q p}-1\right) /\left(e^{q}-1\right)$ for Eq. (16). 
This situation is actually not very special. Consider for instance the more general state

$$
\rho=x\left|\Psi_{0}\right\rangle\left\langle\Psi_{0}|+(1-x)| u v\right\rangle\langle u v|,
$$

where $|u v\rangle=|u\rangle_{A}|v\rangle_{B}$ is an arbitrary separable pure state of the two qubits. This state is again entangled $\forall x>0$, since the partial transpose of $\rho$ has a negative eigenvalue

$$
\sigma_{1}=\frac{1}{2}[1-x-\sqrt{1-2 x(1-x) r}], \quad r=|\langle u \mid v\rangle|^{2},
$$

with $\sigma_{1}=-x(1-r) / 2+O\left(x^{2}\right)$ for $x \rightarrow 0$. On the other hand, the eigenvalues of $\rho$ are

$$
\left(\frac{1}{2}(1+z), \frac{1}{2}(1-z), 0,0\right), \quad z=\sqrt{1-2 x(1-x)(1+r)},
$$

while those of $\rho_{A}, \rho_{B}$ are again $(1-x / 2, x / 2)$. Hence, $p_{1}$ $=(1+z) / 2, p_{1}^{A}=(1-x / 2)$, and $p_{1}>p_{1}^{A}$ only for

$$
x>x_{c}=2 r /(1+2 r) \text {. }
$$

Thus, $S_{f}^{A}(\rho)<0$ if and only if $x_{c}<x<1$, for any concave $f$. Again, the entropic criterion fails to detect entanglement for $0<x<x_{c}$. For $r=1$, we recover the results of the previous example, whereas for $r=0$, i.e., $|u v\rangle=|\uparrow \downarrow\rangle, \sigma_{1}=-x / 2$, and $x_{c}=0$, so that $S_{f}^{A}(\rho)<0 \forall x>0$. This is the only case where the entropic criterion predicts the full interval of inseparability.

Let us still consider the example of Refs. [28,18],

$$
\rho=x|\Psi\rangle\langle\Psi|+(1-x)(\mid \uparrow \uparrow \uparrow\langle\uparrow \uparrow|+| \downarrow \downarrow\rangle\langle\downarrow \downarrow|) / 2,
$$

with $|\Psi\rangle=a|\uparrow \downarrow\rangle+b|\downarrow \uparrow\rangle, \quad|a|^{2}+|b|^{2}=1$. As shown in [18], this state is entangled just for

$$
x>x_{e}=(1+2|a b|)^{-1},
$$

since the lowest eigenvalue of the partial transpose is $\sigma_{1}=[1-x(1+2|a b|)] / 2 \quad$ for $\quad x>\left[2(1+|a b|)-\left.|| a\right|^{2}\right.$ $\left.-|b|^{2} \mid\right]^{-1}$. However, the eigenvalues of $\rho$ are $(x,(1-x)$ $/ 2,(1-x) / 2,0) \quad$ while those of $\rho_{A}, \rho_{B}$ are $\left[1 \pm x\left(|b|^{2}-|a|^{2}\right)\right] / 2$. The largest eigenvalue of $\rho\left(p_{1}=x\right.$ for $x>1 / 3)$ is greater than that of $\rho_{A}\left[p_{1}^{A}=\left(1+x \|\left. a\right|^{2}\right.\right.$ $\left.\left.-|b|^{2} \mid\right) / 2\right]$ only for

$$
x>x_{c}=\left(2-\left.|| a\right|^{2}-|b|^{2} \mid\right)^{-1} .
$$

But $x_{c} \geqslant x_{e}$, with $x_{c}=x_{e}$ just for $|a|=|b|$ or in the trivial separable cases $b=0$ or $a=0$. Hence, if $|a| \neq|b|$ and $a b$ $\neq 0, S_{f}^{A}(\rho)$ will not detect entanglement for $x_{e}<x<x_{c}$. Note also that, for $x>x_{c}, S_{f}^{A}(\rho)$ is in this case not necessarily negative for any $f$, but will become negative for sufficiently large $q$ in the Tsallis case or in Eq. (14) or Eq. (16), as shown in Fig. 2. The value of $x$ where $S_{f}^{A}(\rho)=0$ actually converges exponentially fast to $x_{c}$ for $q \rightarrow \infty$ in Eq. (3) or Eq. (16). This will occur whenever the degeneracies of $p_{1}$ and $p_{1}^{A}$ coincide.

The entropic criterion will provide, however, necessary and sufficient conditions for separability for any density $\rho$ diagonal in the Bell basis [7], i.e., the basis of maximally

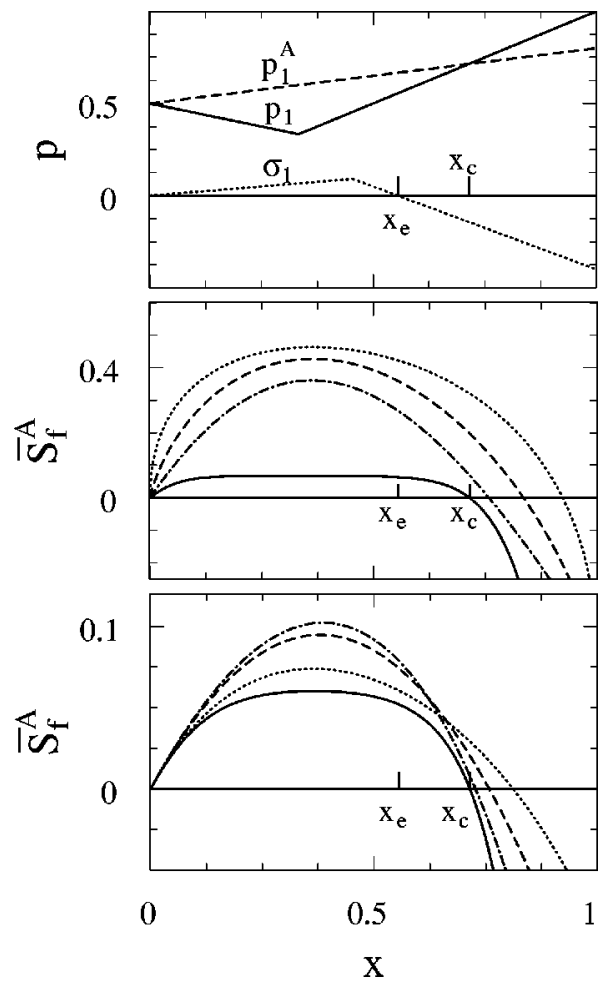

FIG. 2. Same details as Fig. 1 for the density (20) with $|a|^{2}$ $=4 / 5$. The values of $q$ for the different lines in the center and bottom panels are the same as those of Fig. 1.

entangled states $\left|\Psi_{0}\right\rangle,\left|\Psi_{1}\right\rangle=(|\uparrow \downarrow\rangle+|\downarrow \uparrow\rangle) / \sqrt{2},\left|\Psi_{2,3}\right\rangle$ $=(|\uparrow \uparrow\rangle \pm|\downarrow \downarrow\rangle) / \sqrt{2}$. In such a case,

$$
\rho=\sum_{i=0}^{3} q_{i}\left|\Psi_{i}\right\rangle\left\langle\Psi_{i}\right|
$$

is known to be entangled if and only if $p_{1}>1 / 2$ [5], where $p_{1}=\operatorname{Max}\left[\left\{q_{i}\right\}\right]$ is the largest eigenvalue of $\rho$. This may be obtained directly with the Peres criterion, as the partial transpose of $\rho$ has eigenvalues $\frac{1}{2}-q_{i}$. Now, for any pure Bell state $\left|\Psi_{i}\right\rangle\left\langle\Psi_{i}\right|$, the reduced density matrices are fully mixed, with eigenvalues $\left(\frac{1}{2}, \frac{1}{2}\right)$, so that the same will occur for any state of the form (21). The condition $p_{1} \leqslant p_{1}^{A}$ then becomes equivalent, for any state (21), to $p_{1} \leqslant 1 / 2$, i.e., to the necessary and sufficient condition for separability. The entropic criterion will therefore always lead to this condition for $q$ $\rightarrow \infty$ in Eq. (14).

This explains why the entropic criterion for $q \rightarrow \infty$ yields the necessary and sufficient condition for separability for Werner-Popescu states [24,29],

$$
\rho=x\left|\Psi_{0}\right\rangle\left\langle\Psi_{0}\right|+(1-x) I / 4,
$$

where $I=\Sigma_{i=0}^{3}\left|\Psi_{i}\right\rangle\left\langle\Psi_{i}\right|=I_{A} \otimes I_{B}$ is the identity. The eigenvalues of $\rho$ are $p_{1}=(1+3 x) / 4$ and $(1-x) / 4$ (threefold degenerate), and the equation $p_{1} \leqslant \frac{1}{2}$ yields $x \leqslant \frac{1}{3}$, the necessary and sufficient condition [18,30]. Accordingly, for $x>x_{c}$ $=\frac{1}{3}, S_{f}^{A}(\rho)$ will become negative for sufficiently large $q$. The root $x_{r}$ where $S_{f}^{A}(\rho)=0$ will approach $x_{c}$ for $q \rightarrow \infty$, as 


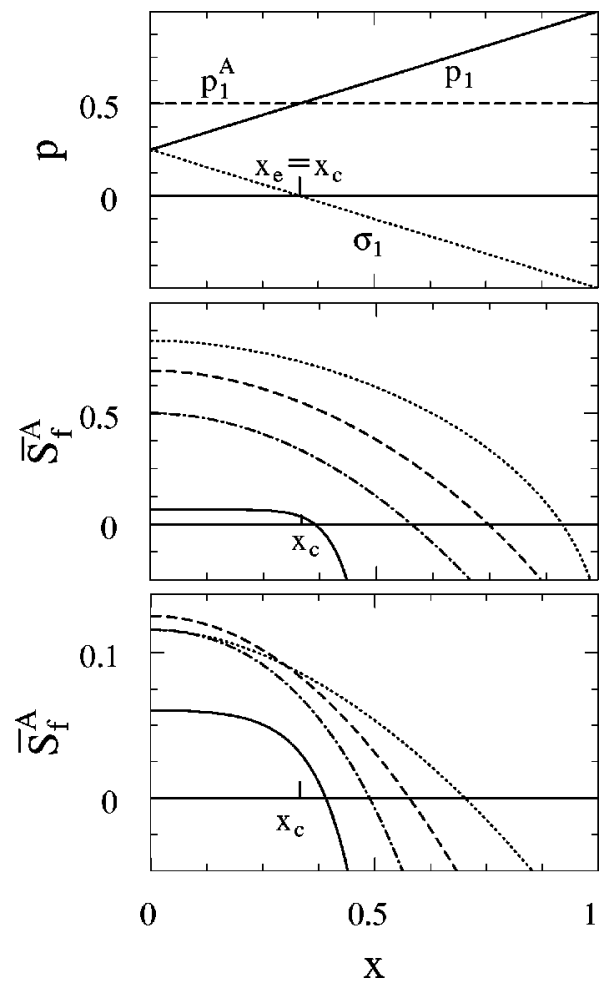

FIG. 3. Same details as Fig. 1 for the density (22). The values of $q$ for the different lines in the center and bottom panels are the same as those of Fig. 1.

seen in Fig. 3, although the convergence is in this case less rapid due to the different degeneracies of $p_{1}$ and $p_{1}^{A}$. For large $q$,

$$
x_{r} \approx \frac{1}{3}+\frac{2 \gamma \ln 2}{3 q}
$$

where $\gamma=1$ for the Tsallis case and $\gamma=2$ for Eq. (16).

Note that the state (20) also becomes of the form (21) for $a= \pm b$ (where the entropic criterion works), as in this case $|\Psi\rangle=\left|\Psi_{0}\right\rangle$ or $\left|\Psi_{1}\right\rangle$ [the remaining term in Eq. (20) is proportional to $\left.\Sigma_{i=2,3}\left|\Psi_{i}\right\rangle\left\langle\Psi_{i}\right|\right]$.

Similar considerations hold for Werner-like states for $n$ qubits [31],

$$
\rho=x|\Psi\rangle\langle\Psi|+(1-x) I / d^{n},
$$

where $d=2,|\Psi\rangle=(|\uparrow \uparrow \cdots \uparrow\rangle+|\downarrow \downarrow \cdots \downarrow\rangle) / \sqrt{2}$ is a maximally entangled state (a Greenberger-Horne-Zeilinger state [32]) and $I$ the identity. The eigenvalues of Eq. (24) are $p_{1}$ $=x+(1-x) / d^{n}$ and $(1-x) / d^{n}\left[\left(d^{n}-1\right)\right.$-fold degenerate $]$. Now, for a subsystem $A_{m}$ with $m$ qubits $(1 \leqslant m \leqslant n-1)$, the reduced density matrix $\rho_{m}$ can be easily shown to have eigenvalues $p_{1}^{m}=x / d+(1-x) / d^{m}(d$-fold degenerate) and (1 $-x) / d^{m}\left[\left(d^{m}-d\right)\right.$-fold degenerate $]$. The necessary condition for separability between the $m$ and $n-m$ subsystems, $p_{1}$ $\leqslant p_{1}^{m}$, leads to

$$
x \leqslant x_{c}^{m} \equiv\left[1+\frac{d^{n-1}(d-1)}{d^{n-m}-1}\right]^{-1},
$$

which is a decreasing function of $m$. The most stringent condition is then obtained for $m=n-1$, i.e., $x \leqslant\left(1+d^{n-1}\right)^{-1}$, which, according to Refs. [31,33], is just the necessary and sufficient condition for full separability. The entropic criterion $S_{f}^{A_{m}}(\rho) \geqslant 0$ will then lead to Eq. (25) for $q \rightarrow \infty$ (as shown in [11] for the Tsallis case). If $d$ is an arbitrary integer $(\geqslant 2)$, the previous discussion and expressions are actually also valid for $n$ qudits ( $n d$-dimensional systems), when $|\Psi\rangle$ is the fully entangled state $\Sigma_{k=0}^{d-1}|k\rangle_{1} \cdots|k\rangle_{n} / \sqrt{d}$ [33].

It should be stressed that for bipartite systems with subsystem dimension $d>2$, the first violation of the majorization relation between $\rho$ and $\rho_{A}$ in an entangled state may also occur for $i>1$ in Eq. (5). For instance, let us briefly discuss the example given in [13], dealing with a system of two identical harmonic oscillators. It was shown that for certain densities $S_{f}^{A}(\rho)$ becomes negative just in a finite interval of $q$ values in the Tsallis case, remaining positive for arbitrary large $q$. This indicates that $\rho$ is not more mixed than $\rho_{A}$, and hence entangled, but still has $p_{1}<p_{1}^{A}$, which ensures that $S_{f}^{A}(\rho)$ remains positive for $q \rightarrow \infty$. The first violation of the inequalities (5) is therefore taking place for $i>1$ (we have verified that this occurred for $i=2$ ). Nevertheless, it should be remarked that in such situations, if $\mathcal{S}_{i}$ is only slightly larger than $\mathcal{S}_{i}^{A}$ and $i>1, S_{f}^{A}(\rho)$ may remain positive for all $q>0$ in the case (3), and is then unable to detect entanglement. The same happens with the entropy (16).

In summary, we have shown that the generalized entropic criterion $S_{f}^{A}(\rho)=S_{f}(\rho)-S_{f}\left(\rho_{A}\right) \geqslant 0$ constitutes, for any concave entropic function $f$, a necessary condition for separability. For $q \rightarrow \infty$ in Eq. (3), or in general Eq. (14), it becomes equivalent to the condition (11) between the largest eigenvalues of $\rho$ and $\rho_{A}$. Nonetheless, the entropic criterion is not a sufficient one in general. We have provided examples of entangled densities of two qubits where $p_{1}<p_{1}^{A}$, in which case $\rho$ remains more mixed than $\rho_{A}$, implying $S_{f}^{A}(\rho) \geqslant 0$ for any choice of entropic function $f$. However, the condition $p_{1}$ $\leqslant p_{1}^{A}$ becomes sufficient in some important cases, which include any density diagonal in the Bell basis in a two-qubit system, and also Werner-like states in $n$-qubit (or qudit) systems. In these cases the inequality $S_{f}^{A}(\rho) \geqslant 0$ will lead, for $q \rightarrow \infty$ in Eqs. (3) or Eq. (14), to the necessary and sufficient condition for separability.

The condition $S_{f}^{A}(\rho) \geqslant 0$ for any concave entropic function $f$ is equivalent to the requirement that $\rho$ be more mixed than $\rho_{A}$, a general necessary condition for separability [25]. Let us remark that this requirement is stronger than the condition $S_{f}^{A}(\rho) \geqslant 0 \forall q>0$ in Eq. (3) [or $\forall q$ in Eq. (16)]. Other families of concave entropic functions are required in general to detect that $\rho$ is not more mixed than $\rho_{A}$ when the first violation of Eqs. (5) occurs for $i>1$, although in many cases this can also be seen with the entropies (3) or (16). In such situations $S_{f}^{A}(\rho)$ will remain positive for $q \rightarrow \infty$ but may become negative at finite values of $q$.

R.R. and N.C. acknowledge support from CIC and CONICET, respectively, of Argentina. 
[1] E. Schrödinger, Naturwissenschaften 23, 807 (1935); Proc. Cambridge Philos. Soc. 31, 555 (1935).

[2] D. P. DiVincenzo, Science 270, 255 (1995); C. H. Bennett, D. P. DiVincenzo, J. A. Smolin, and W. K. Wootters, Phys. Rev. A 54, 3824 (1996).

[3] A. K. Ekert, Phys. Rev. Lett. 67, 661 (1991); Nature (London) 358, 14 (1992).

[4] C. H. Bennett et al., Phys. Rev. Lett. 70, 1895 (1993).

[5] R. Horodecki and M. Horodecki, Phys. Rev. A 54, 1838 (1996); R. Horodecki, P. Horodecki, and M. Horodecki, Phys. Lett. A 210, 377 (1996).

[6] N. J. Cerf and C. Adami, Phys. Rev. Lett. 79, 5194 (1997); Phys. Rev. A 55, 3371 (1997).

[7] R. Horodecki, M. Horodecki, and P. Horodecki, Phys. Rev. A 59, 1799 (1999).

[8] C. Brukner and A. Zeilinger, Phys. Rev. Lett. 83, 3354 (1999).

[9] S. Abe and A. K. Rajagopal, Phys. Rev. A 60, 3461 (1999).

[10] A. Vidiella-Barranco, Phys. Lett. A 260, 335 (1999).

[11] S. Abe and A. K. Rajagopal, Physica A 289, 157 (2001); S. Abe, e-print quant-ph/0104135.

[12] C. Tsallis, S. Lloyd, and M. Baranger, Phys. Rev. A 63, 042104 (2001)

[13] C. Tsallis, D. Prato, and C. Anteneodo, e-print quant-ph/0202077.

[14] F. Giraldi and P. Grigolini, Phys. Rev. A 64, 032310 (2001).

[15] N. Canosa and R. Rossignoli, Phys. Rev. Lett. 88, 170401 (2002)
[16] E. Lieb and M. B. Ruskai, Phys. Rev. Lett. 30, 434 (1973).

[17] A. Wehrl, Rev. Mod. Phys. 50, 221 (1978).

[18] A. Peres, Phys. Rev. Lett. 77, 1413 (1996).

[19] M. Horodecki, P. Horodecki, and R. Horodecki, Phys. Lett. A 223, 1 (1999).

[20] C. Tsallis, J. Stat. Phys. 52, 479 (1988).

[21] R. Rossignoli and N. Canosa, Phys. Lett. A 264, 148 (1999).

[22] C. H. Bennett, H. J. Bernstein, S. Popescu, and B. Schumacher, Phys. Rev. A 53, 2046 (1996).

[23] W. K. Wootters, Phys. Rev. Lett. 80, 2245 (1998).

[24] R. F. Werner, Phys. Rev. A 40, 4277 (1989).

[25] M. A. Nielsen and J. Kempe, Phys. Rev. Lett. 86, 5184 (2001).

[26] A. Rényi, Probability Theory (North-Holland, Amsterdam, 1970).

[27] N. J. Cerf, C. Adami, and R. M. Gingrich, Phys. Rev. A 60, 898 (1999).

[28] N. Gisin, Phys. Lett. A 210, 151 (1996).

[29] S. Popescu, Phys. Rev. Lett. 72, 797 (1994).

[30] C. H. Bennett et al., Phys. Rev. Lett. 76, 722 (1996).

[31] W. Dür, J. I. Cirac, and R. Tarrach, Phys. Rev. Lett. 83, 3562 (1999).

[32] D. M. Greenberger, M. Horne, and A. Zeilinger, in Bell's Theorem, Quantum Theory and Conceptions of the Universe, edited by M. Kafatos (Kluwer, Dordrecht, 1989), p. 69.

[33] A. Pittenger and M. H. Rubin, Phys. Rev. A 62, 032313 (2000). 